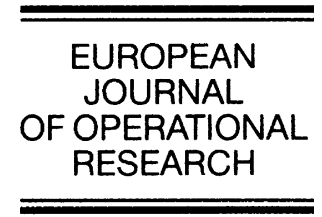

www.elsevier.com/locate/dsw

\title{
On a bi-dimensional dynamic alternative routing method
}

\author{
Lúcia Martins ${ }^{\mathrm{a}, \mathrm{c}, *}$, José Craveirinha ${ }^{\mathrm{a}, \mathrm{c}}$, João N. Clímaco ${ }^{\mathrm{b}, \mathrm{c}}$, Teresa Gomes ${ }^{\mathrm{a}, \mathrm{c}}$ \\ ${ }^{a}$ Department of Electrical and Computer Engineering, Faculty of Sciences and Technology of the University of Coimbra, \\ Pinhal de Marrocos, 3030-290 Coimbra, Portugal \\ ${ }^{\mathrm{b}}$ Faculty of Economics, University of Coimbra, Av. Dias da Silva, 3000 Coimbra, Portugal \\ ${ }^{\mathrm{c}}$ INESC-Coimbra, Rua Antero de Quental 199, 3000-033 Coimbra, Portugal
}

Received 1 September 2002; accepted 1 March 2004

Available online 19 August 2004

\begin{abstract}
The analysis of a bi-dimensional dynamic routing model for alternative routing telecommunication networks led to the identification of an instability problem in the synchronous path selection associated with the complex interdependencies among the coefficients of the objective functions and the computed paths for every node pair. In this paper an analytical model enabling to make explicit this problem and evaluate its effects in terms of two global network criteria, is presented. Also a heuristic procedure dedicated to overcome this instability problem and select "good" compromise solutions in terms of network performance is developed. Finally the performance of the proposed routing method using the heuristic is compared by recurring to discrete-event simulation with a reference dynamic routing method (Real Time Network Routing) for some test networks.
\end{abstract}

(c) 2004 Elsevier B.V. All rights reserved.

Keywords: Bi-criteria analysis; Routing; Heuristics; Telecommunications

\section{Introduction and background}

Routing is a key element of any telecommunications network functional structure and has a deci-

\footnotetext{
${ }^{*}$ Corresponding author. Address: Department of Electrical and Computer Engineering, Faculty of Sciences and Technology of the University of Coimbra, Pinhal de Marrocos, 3030290 Coimbra, Portugal.

E-mail address: lucia@deec.uc.pt (L. Martins).
}

sive impact on network performance and cost. A routing method is primarily concerned with the determination and selection of a path or set of paths between every pair of nodes of the network representation, seeking to optimise certain objective(s) and satisfy certain constraints of a technical nature. In the formulation of the various routing problems in telecommunication networks these may be modelled through teletraffic networks the description of which involves the following elements: a graph $(V, L)$ defining the network 
topology where the nodes $v \in V$ may represent switches, exchanges (groups of switches with known architecture) servers or routers and the arcs (or links) $l \in L$ represent transmission facilities; the capacities of the arcs expressed in terms of bandwidth or equivalent number of transmission channels (usually based on multiples of $64 \mathrm{~kb} / \mathrm{s}$ ); the node-to-node traffic flows which in general may be modelled as stochastic marked point processes (enabling to represent the instants of call arrivals, their duration, bandwidth requirements or other technical requirements); the routing principle specified in terms of the essential features of the routing function (in the network), e.g. whether it is static or dynamic and the number of paths which may be attempted by a call of any given traffic flow or the maximum number of links per path. A routing method can be defined as a particular specification of a certain routing principle, including as key element the algorithm or set of rules which enables to perform the path computation and path selection for every traffic flow having in mind the objective(s) and requirements of the underlying routing principle.

The evolution of multiservice telecommunications network functionalities leads to the necessity of dealing with multiple, fine grain and heterogeneous quality of service requirements which will have to be reflected in some manner in the routing problem formulation. When applied to routing methods this concern led to a new routing concept designated as QoS (Quality of Service) routing which involves the selection of a chain of network resources satisfying certain QoS requirements and seeking simultaneously to optimise the route associated metric(s) (or a sole function of different metrics) such as cost, delay, number of hops or blocking probability. This trend implies considering explicitly distinct metrics in routing algorithms such as in references [22,23] or [20]. In this context the path selection problem was normally formulated as a shortest path problem with a single objective function, either a single metric or encompassing different metrics. QoS requirements were then incorporated into these models by means of additional constraints and the path selection problem (or routing problem in a strict sense) was solved by resorting to different types of heuristics usually based on Dijkstra or Bellman-Ford shortest path algorithms.

Therefore there are potential advantages in modelling the routing problem of this type as a multiple objective problem. Multiple objective routing models enable to grasp the trade-offs among distinct QoS requirements by enabling to represent explicitly, as objective functions, the relevant metrics for each traffic flow and treating in a consistent manner the comparison among different routing alternatives.

On the other hand, the utilisation of dynamic routing (i.e. a routing principle involving the calculation of time-variant paths or path sets between every pair of nodes as a function of relevant measurable network functioning characteristics) in various types of networks is well known to have a quite significant impact on network performance and cost, namely considering time-variant traffic patterns, overload and failure conditions (see for example [12] and [4]).

In the case of circuit-switched alternative routing networks any call of traffic flow $f$ from node $v_{i}$ to node $v_{t}$ may attempt paths $r^{1}(f)$, $r^{2}(f), \ldots, r^{M}(f)$ in this order; the first path (or route) with at least one free channel (channel is here defined as the amount of arc capacity required to carry a call of flow $f$ ) in every of its arcs and satisfying other possible requirements of the routing method will be the one to be used by the call; if none of those paths satisfies these conditions the call is lost, the associated probability being designated as marginal blocking probability (or call congestion) for traffic flow $f$. In alternative dynamic routing methods the traffic flexible carrying capacity of alternative routing is associated with the adaptive nature of dynamic routing by enabling the network routing to react in an effective manner (with respect to pre-defined criteria) to dynamic changes in traffic intensities, namely in overload conditions, and to failure states in the links and/or nodes of the network. These methods are the most efficient (albeit complex) type of routing methods conceivable for these networks.

In a previous paper [9] the authors presented the essential features of a 'multiple objective dynamic alternative routing method' (MODR in short) of 
periodic state-dependent routing type, based on a bi-objective shortest path model for solving a static alternative routing problem, focused on the calculation and selection of two paths $(M=2)$ (first and second choice routes) for every traffic flow, assuming fixed node-to-node traffic intensities and fixed values of the two objective function coefficients. In its initial formulation for multiexchange circuit-switched networks the model uses implied costs and blocking probabilities as metrics for the path calculation problem. In other network environments in terms of underlying technologies and supplied services other QoS metrics can be easily integrated in this type of routing model.

In [18] the authors have identified an instability problem in the synchronous path selection procedure, associated with the bi-objective shortest path calculations for every pair of nodes, in successive path updating periods. This instability problem is associated with the complex non-linear interdependencies between the coefficients of the two objective functions, with the interdependencies between both sets of coefficients and the computed paths and from the discrete nature of the model. This phenomena is expressed by the fact that the paths computed for every node pair in each time period tend to oscillate between a few sets of solutions some of which may lead to poor global network performance. Note that even using a single-objective formulation of the adaptive alternative-routing problem, the corresponding optimal routing problem can be shown to be NP-complete in the strong sense (see [10]) which is an indication of computational intractability even for near-optimal solutions.

In order to cope with the complexity of the proposed routing model this paper describes a complete analytical model enabling to make it explicit the instability problem in the context of a bi-dimensional alternative dynamic routing model and calculate two relevant global network performance criteria. Also a heuristic procedure enabling to overcome this instability problem and select "good" compromise routing solutions, is presented together with a simulation study for relative performance evaluation of the proposed routing method incorporating this heuristic. The paper is organised as follows. Section 2 reviews the main features of the MODR method under analysis. Section 3 presents a complete analytical model and a bi-dimensional compromise dynamic alternative routing problem at the global network level; this enabled to make it explicit the relations and interdependencies among the various mathematical entities. Section 4, taking as basis relevant requirements [18], presents the formalisation of a heuristic of synchronous path selection enabling to select "good" compromise solutions in terms of the two global network performance criteria. Section 5 analyses the relative performance of the routing method using the proposed heuristic procedure by recurring to a discrete event simulation platform enabling to compare the global network performance criteria values of the model, incorporating the heuristic, with those obtained with a reference dynamic routing method RTNR (Real-Time Network Routing) developed by AT\&T, for some test networks. Finally some conclusions and further developments of this work will be discussed.

\section{General features of the MODR method}

The 'multiple objective dynamic routing method' MODR proposed in [9] is a new type of periodic state dependent routing method (i.e. it uses a dynamic routing principle where the paths change periodically as a function of some measure of the network functioning state) based on a multiple dimension routing paradigm. In its general formulation MODR has the following main features: (i) paths which may be used by calls between every node pair (up to $M=2$ in the present implementation) can change periodically as a function of periodic updates of certain QoS related parameters obtained from real-time measurements; (ii) the calculation of paths is based on a bi-objective shortest path model (reviewed in Section 3) which uses in the present formulation (for loss traffic) as path metrics to be minimised, implied costs (in the sense defined by Kelly [15]) and blocking probabilities; (iii) the parameters obtained from periodical measurements in the network are reflected directly in the calculation of the coefficients of the objective function of the bi-objective shortest path model; (iv) a very efficient algorithmic approach (designated as 
MMRA) is used to tackle this bi-objective shortest path model which enables to calculate a first and second choice routes for each node-to-node traffic flow, by recurring to an extremely efficient $k$-shortest path algorithm and to the definition of preference regions in the objective functions space; (v) the boundaries of the preference regions, which correspond to required or acceptable values for each path metric depend on the type of node-tonode traffic flow (concerning the associated type of network service) and vary dynamically hence reflecting the network working conditions.

As for the way in which the paths are selected in the MODR method, the first path is always the direct route whenever it exists. The remaining routes for traffic flows between an exchange pair are selected from the MMRA, taking into account the defined priority regions.

In order to cope with the complexity of the MODR method and make it explicit the relations between the various entities that intervene in the method, a complete network analytical model is developed in the next section. This will enable to formulate a bi-dimensional network dynamic alternative routing problem where one seeks a compromise solution between two network performance metrics. This modelling framework also permits to understand the instability problem mentioned in the introductory section and justify the proposal and specification of a heuristic of synchronous path selection. The aim of the heuristic is to select a "good" compromise solution for the network problem, corresponding to a set of $M$ ordered paths for every node pair, which guarantees some acceptable trade-off in terms of the used network metrics, namely mean network blocking probability and maximal node-to-node blocking probability. The heuristic uses MMRA as a core sub-routine which tackles the auxiliary bi-objective shortest path problem. It is important to note that the whole procedure of path calculation and path selection will have to run automatically in the framework of a routing control mechanism (see [9]) hence excluding the use of interactive approaches in the resolution of the associated decision problems. This has natural implications in the adopted resolution procedures, as discussed in the next section.

\section{Analytical model}

We begin by formalising a bi-dimensional alternative dynamic network routing problem which expresses the network decision problem that MODR has to address through an automatic calculation procedure of periodic type.

\section{Notation:}

- $G=(V, L)$ - undirected graph representing the network topology where $V$ is the node set and $L$ the arc set;

- $f \equiv\left(v_{s}, v_{t}, \gamma\right) ; \quad v_{s}, v_{t} \in V ; \quad v_{s} \neq v_{t}$-traffic flow from node $v_{s}$ to node $v_{t}$ where $\gamma$ represents a traffic descriptor that in general includes all characteristics which enable to define completely the associated stochastic process (e.g. arrival intensity $\lambda_{f}$, call mean service time $h_{f}$, number $n_{f}$ of channels required by each call in every arc of each attempted path); in the present formulation for single channel traffic $n_{f}=1$ and $\gamma$ is reduced to the value of the traffic offered in Erlangs, $A_{t}(f)$ (defined below);

- $\mathscr{F}$ - set of all traffic flows in the network;

- $A_{t}(f)=\lambda_{f} h_{f}$-traffic offered (in Erlangs) for traffic flow $f=\left(v_{i}, v_{j}, \gamma\right) \in \mathscr{F}$ at time $t=$ $n T(n=1,2, \ldots)$ the matrix $\left[A_{t}(f)\right]_{i, j}$ is denoted by $\bar{A}_{t}$;

- $B(f)$ - call blocking probability for traffic flow $f \in \mathscr{F}$ also designated as marginal blocking probability (i.e. the probability of a call of flow $f$ being blocked in the network);

- $\mathscr{P}_{f}$ — set of all loopless paths in $G$, from $v_{s}$ to $v_{t}$, for flow $f=\left(v_{s}, v_{t}, \gamma\right)$;

- $R_{t}(f)=\left\{r^{1}(f), r^{2}(f): r^{1}(f), r^{2}(f) \in \mathscr{P}_{f} \wedge\right.$ $\left.\left|r^{i}(f)\right|<D(i=1,2)\right\}$ - ordered set of paths (or routes) which may be used by flow $f$ at time $t$; a constraint $D$ on the maximum number of links per path is also introduced as a feature of the routing method (in fully meshed networks usually $D=2$, cf. [4]);

$\bar{R}_{t}=\left\{R_{t}\left(f_{1}\right), \ldots, R_{t}\left(f_{|\mathscr{F}|}\right)\right\}$ is the total route set that represents the routing plan for all flows $f$ at time $t$.

- $\lambda_{r^{i}(f)}$-marginal traffic carried in route $r^{i}(f)$;

- $B_{k}$-average blocking probability experienced by a call on link $l_{k}=\left(v_{i}, v_{j}\right) \in L$; 
- $C_{k}$ - capacity of link $l_{k}=\left(v_{i}, v_{j}\right) \in L$; the capacity vector is denoted by $\bar{C}=\left[C_{k}\right]$;

- $c_{k}$-implied cost associated with the acceptance of a call on link $l_{k}$ (defined through Eq. (11)); the implied cost vector is denoted by $\bar{c}=\left[c_{k}\right]$;

- $\rho_{k}$-total offered traffic to link $l_{k}$ (the mean of the total number of calls offered to $l_{k}$ during the call mean service time);

- $R_{k}=\left\{r(f) \in R_{t}\left(f_{1}\right) \cup \ldots \cup R_{t}\left(f_{|F|}\right): l_{k} \in r(f)\right\}$ - set of routes (of all traffic flows) which may use link $l_{k}$, at time $t$;

- $L_{r^{i}(f)}$-call blocking probability on route $r^{i}(f)$.

Assuming all traffic flows are homogeneous Poissonian and independent, negative exponentially distributed service times, statistical independence in the occupations of the links and node disjoint routes $r^{1}(f), r^{2}(f)$ for each traffic flow $f$ one may write

$$
\begin{aligned}
\rho_{k}= & \sum_{f: l_{k} \in r^{1}(f)} A_{t}(f) \prod_{l_{j} \in r^{1}(f)-\left\{l_{k}\right\}}\left(1-B_{j}\right) \\
& +\sum_{f: l_{k} \in r^{2}(f)} A_{t}(f) L_{r^{1}}(f) \prod_{l_{i} \in r^{2}(f)-\left\{l_{k}\right\}}\left(1-B_{i}\right), \\
B_{k}= & E\left(\rho_{k}, C_{k}\right), \\
L_{r^{i}(f)} & =1-\prod_{l_{j} \in r^{i}(f)}\left(1-B_{j}\right) \quad(i=1,2),
\end{aligned}
$$

where $E(A, C)$ is the Erlang B function for traffic offered $A$ and $C$ channels which gives the blocking probability on a $\mathrm{M} / \mathrm{M} / \mathrm{C}$ loss system

$E(A, C)=\frac{A^{C}}{C !}\left[\sum_{i=0}^{C} \frac{A^{i}}{i !}\right]^{-1}$.

For given $\bar{A}_{t}, \bar{C}$ and $\bar{R}_{t}$, the blocking probabilities $B_{k}$ can be computed by resolving numerically the system of non-linear equations (1)-(3) in $B_{k}$ $(k=1,2, \ldots,|L|)$. Note that $B_{k}$ depends on $\bar{R}_{t}$ via $R_{k}$. Also note that the used assumptions concerning the Poissonian nature of the traffic flows and the condition that the routes $r^{1}(f), r^{2}(f)$ are disjoint (usually assumed in this type of model) could be easily relaxed at the cost of adding further complexity to the system of equations enabling to express $B_{k}$ in terms of $\bar{A}_{t}, \bar{C}$ and $\bar{R}_{t}$ (see e.g. in [8]).
Now a network bi-dimensional dynamic alternative routing problem can be formulated by considering that the first objective in terms of network performance is the maximisation of the total traffic carried in the network $A_{c}$ (mean value of the total number of calls carried by the network at any given period $t$ ), which is the objective in all "classical" single-objective routing models. This is equivalent to minimising the network mean blocking probability $B_{m}$ (mean blocking probability for any call offered to the network). The second objective, proposed by the authors in [18] is the minimisation of the maximal marginal blocking probability $B_{M}$. All these quantities may be expressed in terms of the call congestions $B(f)$ for all $f \in F$ and the total traffic offered

$A_{t}^{o}=\sum_{f \in F} A_{t}(f)$.

Therefore, for given $G, \bar{A}_{t}, \bar{C}$, one may formalise a network dynamic alternative routing problem as a bi-dimensional compromise decision problem at network performance level, in the decision variables $\bar{R}_{t}$.

$$
\begin{aligned}
& \text { (Problem } \mathscr{P}_{G}^{(2)} \text { ) } \\
& \min _{\bar{R}_{t}} B_{m}=\sum_{f \in \mathscr{F}} \frac{A_{t}(f) B(f)}{A_{t}^{0}}, \\
& \min _{\bar{R}_{t}} B_{M}=\max _{f \in \mathscr{F}}\{B(f)\}, \\
& \text { s.t. } \quad B(f)=L_{r^{1}(f)} L_{r^{2}(f)} \\
& \quad \text { and equations }(1)-(3) .
\end{aligned}
$$

It has been proved in [10] that, assuming quasistationary conditions in successive route updating periods, such that the offered traffic stochastic features remain stationary during periods which are relatively long compared to the solution time, the single-objective adaptive alternative routing problem (corresponding to the o.f. (6)) is NP-complete in the strong-sense, even in the "degenerated" simpler case where $M=1$ (no alternative route provided). Note that our model is a bi-objective formulation of this type of problem. Taking further into account the nature and the interdepend- 
encies between the functions $B_{m}(6)$ and $B_{M}(7)$ and their dependency on the routing plan solutions $\bar{R}_{t}$ (via the $\{B(f)\}$ given by (8) and (1)-(3)) - these are strong indications of extreme intractability of the network problem $\mathscr{P}_{G}^{(2)}$.

As for the possible conflict between the objective functions in $\mathscr{P}_{G}^{(2)}$ it can be said that in many situations, in alternative routing networks, the minimisation of $B_{m}$ is associated with a deterioration on $B(f)$ for "small" intensity traffic flows $A_{t}(f)$, leading to an increase in $B_{M}$. In conventional single-objective routing models this effect is usually limited by imposing upper bounds on $B(f)$.

The basis of the procedure for tackling this very complex network problem relies on the formulation of a bi-objective shortest path problem for every traffic flow $f=\left(v_{s}, v_{t}, \gamma\right)$, where the objective functions are path implied cost and blocking probability

$$
\begin{aligned}
& \text { (Problem } \left.\mathscr{P}^{(2)}\right) \\
& \min z^{n}=\sum_{l_{k}=\left(v_{i}, v_{j}\right) \in L} \mathscr{C}_{k}^{n} x_{i j} \quad(n=1,2)
\end{aligned}
$$

s.t.

$$
\begin{aligned}
& \sum_{v_{j} \in V} x_{s j}=1, \\
& \sum_{v_{i} \in V} x_{i j}-\sum_{v_{q} \in V} x_{j q}=0 \quad \forall v_{j} \in V, \quad\left(v_{j} \neq v_{s}, v_{t}\right), \\
& \sum_{v_{i} \in V} x_{i t}=1, \\
& x_{i j} \in\{0,1\} \quad \forall l_{k}=\left(v_{i}, v_{j}\right) \in L, \\
& x_{i j}=1 \quad \text { if } l_{k}=\left(v_{i}, v_{j}\right) \text { belongs to the path; } \\
& x_{i j}=0 \text { otherwise, }
\end{aligned}
$$

where

$\mathscr{C}_{k}^{1}=c_{k} \quad$ and $\quad \mathscr{C}_{k}^{2}=-\log \left(1-B_{k}\right)$.

The log is used to transform blocking probability into an additive metric.

The implied cost $c_{k}$ associated with link $l_{k}$ is a powerful concept in telecommunication routing theory, due to Kelly [15] and represents the expected value of the increase in lost calls (on all routes of all traffic flows using $l_{k}$ ) as a result of accepting a call of a given traffic flow, on arc $l_{k}$.
Under the same assumptions which we used in (1)-(4) it follows, according to [15]:

$$
\begin{gathered}
c_{k}=\eta_{k}\left(1-B_{k}\right)^{-1}\left[\sum_{f: l_{k} \in r^{1}(f)} \lambda_{r^{1}(f)}\left(s_{r^{1}(f)}+c_{k}\right)\right. \\
\left.+\sum_{f: l_{k} \in r^{2}(f)} \lambda_{r^{2}(f)}\left(s_{r^{2}(f)}+c_{k}\right)\right], \\
s_{r^{2}(f)}=w(f)-\sum_{l_{j} \in r^{2}(f)} c_{j}, \\
s_{r^{1}(f)}=w(f)-\sum_{l_{j} \in r^{1}(f)} c_{j}-\left(1-L_{r^{2}(f)}\right) s_{r^{2}(f)},
\end{gathered}
$$

where $w(f)$ is the expected revenue for an accepted call of traffic flow $f, s_{r^{i}}(f)$ is the surplus value of a call on route $r^{i}(f)$ and $\eta_{k}$ is the increase in the blocking on the link $l_{k}$ originated by a unit decrease in the arc capacity:

$$
\begin{aligned}
& \eta_{k}=E\left(\rho_{k}, C_{k}-1\right)-E\left(\rho_{k}, C_{k}\right) \\
& \text { and } \\
& \lambda_{r^{1}(f)}=A_{t}(f) \prod_{l_{j} \in r^{1}(f)}\left(1-B_{j}\right), \\
& \lambda_{r^{2}(f)}=A_{t}(f) L_{r^{1}(f)} \prod_{l_{j} \in r^{2}(f)}\left(1-B_{j}\right) .
\end{aligned}
$$

The use of the solutions to the problem $\mathscr{P}^{(2)}$ obtained from the algorithmic approach MMRA (discussed in $[9,18]$ ) as a basis for constructing the procedure which seeks compromise solutions for the network problem $\mathscr{P}_{G}^{(2)}$ is justified by the property that minimising $z^{1}$ in $\mathscr{P}^{(2)}$ tends to minimise $B_{m}$, when searching for a path for flow $f$ assuming all the remaining conditions in the network (namely the routes assigned to all other flows and all the link implied costs) were maintained constant while the minimisation of $z^{2}$ in $\mathscr{P}^{(2)}$ tends to achieve the minimisation of $B_{M}$, under similar assumptions [18].

From the previous equations (1)-(4) (concerning $B_{k}$ ) and from Eqs. (11)-(16) one can define implicitly a system of non-linear equations in $B_{k}$ and $c_{k}$ :

$$
\left\{\begin{array}{l}
B_{k}=\beta_{k}\left(\bar{B}, \bar{C}, \overline{A_{t}}, R_{k}\right) \\
c_{k}=\alpha_{k}\left(\bar{c}, \bar{B}, \bar{C}, \overline{A_{t}}, R_{k}\right) \\
(k=1,2, \ldots,|L|)
\end{array}\right.
$$


It should be emphasised the interdependencies between $\left\{c_{k}\right\}$ and $\left\{B_{k}\right\}$ and between these two sets of parameters which define the coefficients of the objective functions and the current total route set $\bar{R}_{t}$, via $R_{k}$.

Of course, from (S1) and the previous analysis on problem $\mathscr{P}_{G}^{(2)}$ overall complexity it is clear that the assumptions that "all remaining conditions in the network are maintained constant", do not hold, which explains the unstable behaviour of the MMRA solutions.

As for the algorithmic approach (MMRA) $[9,18]$ used to calculate solutions for problem $\mathscr{P}^{(2)}$ it is a variant of the algorithm proposed in [1] for a bi-objective shortest path problem of the same type, adapted to the requirements and specificities of MODR. This is now briefly reviewed.

The resolution approach proposed in [1] is inspired by the one presented in [21] and [7] in the framework of a procedure enabling to search interactively non-dominated supported and unsupported paths. It should be stressed that the calculation of the routing plans is supposed to run in an automatic manner, in the framework of a routing control mechanism. The procedure satisfies this requirement integrating the use of a $k$-shortest paths algorithm [17], together with new devices designated by soft constraints (that is constraints not directly incorporated into the mathematical model). In resume, in this approach a specialised automatic algorithmic was developed to obtain non-dominated solutions, which takes into account the specific aspects of a routing problem in a multiservice environment. The main features of this algorithm approach are: (i) to enable that QoS requirements may be expressed as soft constraints on the objective function values in terms of requested and acceptable thresholds for each metric; (ii) the addition of this type of soft constraints defines priority regions, in which nondominated solutions are searched for according to the underlying QoS thresholds (so, the preferences are stated through the soft constraints); (iii) the auxiliary objective function which is used to search for non-dominated solutions is a weighted sum of the two original objective functions, where the weights are arbitrary values between 0 and 1 with sum equal to one (in the present version we used equal weights); (iv) the non-dominated solutions (including those in the interior of the convex hull of the feasible solution set) are computed by means of an extremely efficient $k$-shortest path algorithm proposed in [17] designated as MPS algorithm. It must be noted that in the calculation of non-dominated solutions, namely unsupported non-dominated solutions, it seems useful considering reference point approaches. However, in the case of shortest-path problems, the development of an extremely efficient algorithm (the MPS algorithm [17]) for the $k$-shortest path problem together with the test of non-dominance in [7], creates the possibility of developing very efficient techniques for calculating supported and unsupported non-dominated solutions in this particular context.

MMRA follows the same procedures as in the reviewed approach with the exception of the following points: (i) the sub-algorithm used for calculating $k$-shortest paths it is new variant [13] of the MPS algorithm [17] which enables to calculate in a very efficient manner $k$-shortest paths with a maximal number (typically $D \geqslant 2$ ) of arcs per path hence incorporating the required constraint on the length of paths $r^{1}(f), r^{2}(f)$; (ii) the procedure for solution selection may accept, in certain situations, dominated solutions corresponding to a second-choice route $r^{2}(f)$, namely when such solution is dominated by the one corresponding to $r^{1}(f)$ but is not dominated by any other solution and it is situated in a higher priority region in relation to any other non-dominated solution different from $r^{1}(f)$; (iii) the boundaries of the preference regions (corresponding to acceptable and required values for each o.f.) vary dynamically in order to adapt to variable network loading conditions according to a scheme more flexible and efficient than the one in [9], which is described in Section 4.3.

Other important elements of the resolution of the analytical model are a fixed point iterative scheme enabling the numerical computation of $\bar{B}$ and a similar fixed point iterator to calculate $\bar{c}$ given $(V, L), \bar{C}, \bar{A}_{t}$ and $\bar{R}_{t}$ (therefore all $R_{k}$ are also known), which resolve the systems (S1a) and (S1b), respectively, in this order. The convergence of these numerical procedures designated hereafter as fixed point iterators (or simply, iterators) is 
guaranteed in most cases of practical interest according to $[14,15]$. Supposing that the algorithm MMRA calculates $\bar{R}_{t}$ at every period $t=n T(n=1,2, \ldots)$ where $T$ is the path updating period, the functional interdependencies between the mathematical entities involved in the MODR would be expressed through

- $\bar{R}_{t_{0}}=\bar{R}_{0}$,

- recalculate $\bar{c}, \bar{B}$ with the iterators for previous $\bar{R}_{t}$,

- $\bar{R}_{t}=\operatorname{MMRA}(\bar{c}, \bar{B})$,

where $\bar{R}_{0}$, the initial route set, should be defined from a suitable network dimensioning method, such as in [5], for given nominal traffic matrix $\bar{A}_{0}$. These interdependencies may be illustrated through the diagram in Fig. 1, taking into account (S1a), (S1b) and the expressions defining $B_{m}(6), B_{M}(7)$ and $B(f)(8)$ in terms of $L_{r^{i}}(f)$ (given by (3)). Having in mind these interdependencies the authors have identified in [18] an instability problem in the synchronous path selection procedure that would be obtained through direct application of MMRA for every pair of nodes in successive path updating periods. To tackle this instability problem and seek 'good' compromise solutions for the network problem $\mathscr{P}_{G}^{(2)}$ the heuristic, discussed and formalised in the next section, was developed.

\section{Heuristic for path selection}

\subsection{Path instability}

As a result of the analysed interdependencies among key mathematical entities of the model and the inherent complexity of the global problem $\mathscr{P}_{G}^{(2)}$ it could be expected that iterative application

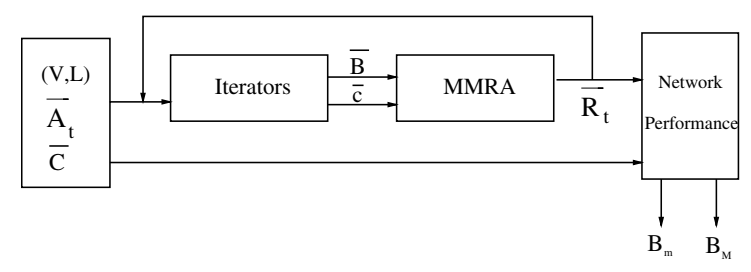

Fig. 1. Functional relations in the MODR model. of the bi-objective algorithmic approach MMRA would generate unstable solutions, possibly leading to poor network performance (under the bi-dimensional model $\left.\left(B_{m}, B_{M}\right)\right)$. In fact such procedure (involving the determination by MMRA of the "best" compromise alternative paths for all origin-destination node pairs as a function of the network state) leads to situations where certain links or paths which were "best" candidates according to the MMRA working, will be in the following path calculation iteration, in a "bad" condition as soon as they are selected as paths of a significant number of $\mathrm{O}-\mathrm{D}$ pairs. This behaviour leads typically to situations where paths chosen by the routing calculation system may oscillate between a few sets of solutions such that in a certain iteration certain links will be very loaded (i.e. they will contribute to many paths) while others are lightly loaded and in the following iteration the more loaded and the less loaded links will reverse their condition. This is a new and specific "bi-objective" case of the known instability problem in single objective adaptive shortest path routing models of particular importance, for example in packet switched networks (see for example [6, Chapter 5]). Instability phenomena are also known in some single-objective alternative dynamic routing models, as analysed in [16] and [11], and may lead, in specific critical situations, to a cascade instability associated with implied costs tending to infinite.

This path instability phenomena in the context of MODR was extensively analysed in [18] and has the following main features: (i) there is a significant range of variation in the values of $B_{m}$ and $B_{M}$ for each traffic overload factor thereby confirming the instability and potential inefficiency of the solutions; (ii) the MMRA solutions correspond in most cases to intermediate values in comparison with the approximations to values of $\min B_{m}$ and $\min B_{M}$ obtained from the corresponding shortest path models, (min of $z^{1}$ and $\min z^{2}$, respectively) as should be expected.

\subsection{A heuristic for synchronous path selection}

A heuristic is now developed for selecting path sets $\bar{R}_{t}(t=n T ; \quad n=1,2, \ldots)$ capable of guaranteeing a good compromise solution in terms of the 
two global network performance criteria $\left(B_{m}, B_{M}\right)$, at every updating period. The foundation of this procedure is to search for the subset of the alternative path set

$\bar{R}_{t-T}^{a}=\left\{r^{2}(f), f \in \mathscr{F}\right\}$

the elements of which should possibly be changed in the next updating period, seeking to minimise $B_{m}$ while simultaneously not letting that smaller intensity traffic flows be affected by excessive blocking probability $B(f)$. Extensive experimentation and analysis of the model led us to propose in [18] the following criterion for choosing candidate paths for possible improvement, which depends explicitly both on the first choice path $r^{1}(f)$ (which in MODR is the direct arc from origin to destination whenever it exists) and on the alternative path $r^{2}(f)$ :

$\xi(f)=F_{1} F_{2}=\left(2 C_{r^{1}(f)}^{1}-C_{r^{2}(f)}^{1}\right)\left(1-L_{r^{1}(f)} L_{r^{2}(f)}\right)$,

$C_{r^{i}(f)}^{1}=\sum_{l_{k} \in r^{i}(f)} c_{k}$.

The objective expressed by the factor $F_{1}$ is to favour (with respect to the need to change the second route) the flows for which the second route has a high implied cost and the 1st route a low implied cost. The factor 2 of $C_{r^{1}(f)}^{1}$ was introduced for normalising reasons taking into account that $r^{1}(f)$ has one arc and $r^{2}(f)$ two arcs, in the considered fully meshed networks. In a more general case where $r^{1}(f)$ has $n_{1}$ arcs and $r^{2}(f) n_{2}$ arcs $\left(n_{1} \leqslant n_{2}\right)$ :

$F_{1}=\left(n_{2}-n_{1}\right) c_{1}^{\prime}+C_{r^{1}(f)}^{1}-C_{r^{2}(f)}^{1}$,

$c_{1}^{\prime}$ being the average implied cost of the arcs in $r^{1}(f)$. The second factor $F_{2}$ expresses the objective of favouring the flows with worse end-to-end blocking probability. The second point to be addressed in the heuristic procedure is to specify how many and which of the second choice routes $r^{2}(f)$ with smaller value of $\xi(f)$ should possibly be changed by applying MMRA once again. In any case, among the recalculated routes only those which lead to solutions which dominate previous ones (in terms of $B_{m}$ and $B_{M}$ ) are finally selected by the procedure as routes to be changed in each path updating period. This requires that the effect of each candidate route, in terms of network performance, be previously anticipated by solving the corresponding analytical model.

The proposed heuristic procedure uses two variables, Npaths and Mpaths that define the current number of candidate paths for improvement in the two main cycles of the heuristic. Mpaths is initialised to the total number of node pairs. Npaths is used in an internal cycle where one seeks to obtain new alternative paths able of improving $B_{m}$ and $B_{M}$ while Mpaths controls an external cycle where Npaths is re-initialised. A variable Ncycle guarantees that the internal cycle (search for minimal $B_{m}$ ) is executed twice, by re-initialising the values of Npaths; in most cases one execution of the cycle was shown to be sufficient for improving $B_{m}$ and more than two cycles would serve no purpose as a result of the oscillatory behaviour of the solution set. Also note that the solution found in the inner cycle depends on the number of routes which one seeks to change and on the initial route set.

Another mechanism introduced in the MODR heuristic is a specific "service protection scheme", aimed at preventing excessive network blocking degradation in overload situations, associated with the utilisation of alternative routes for all node-tonode traffic flows. This mechanism designated as Alternative Path Removal (APR) is based on the elimination of the alternative paths of all traffic flows for which the value of the scalar function $z$ (a convex combination of $z^{1}$ and $z^{2}$ ) involved in the algorithm approach for the bi-objective shortest path model $\mathscr{P}^{(2)}$ is greater than or equal to a certain parameter $z_{\mathrm{APR}}$. Initially this parameter will have to be carefully "tuned" by performing a previous analytical evaluation of network performance and represents a practical absolute threshold above which the use of alternative routing is no longer justified. Now this mechanism is integrated in the heuristic in step (5(f)viD) enabling that $z_{\mathrm{APR}}$ adapts dynamically to overload conditions. The initial value of $z_{\text {APR }}$ was defined empirically taking into account typical ranges of the values $c_{k}$ and $B_{k}$, and is equal to one. The decrease in $z_{\mathrm{APR}}$ only may occur in the second internal cycle of the heuristic and up to a value $M_{\text {sup }}$ of Mpaths since the application of the APR mecha- 
nism for greater values of Mpaths may prevent the consideration as candidate paths of "good" solutions in the initial iterations of the external cycle, which could be particularly negative in low overload conditions. Note that typically the final selected solution is obtained in most cases in the two initial iterations of the external cycle, for the test networks.

Next the heuristic is formalised.

\subsubsection{Heuristic for path selection (MODR-1)}

Denote, for $t=n T(n=1,2, \ldots): \bar{R}_{0}^{(n)}$ the initial set of alternative paths, for which $\bar{B}, \bar{c}$ are the corresponding link metrics, $B_{m}$ and $B_{M}$ the network performance metrics and $N=|V|$, the number of nodes. One will also consider the current set of alternative paths, $\bar{R}^{*}$, for which, in a given iteration $B_{m}$ and $B_{M}$ are both minima, which means that it dominates (or is equal to) the initial path set solution, and the current set $\bar{R}^{a}$ of alternative paths to be tested.

1. $\bar{R}^{*} \leftarrow \bar{R}_{0}^{(n)}, \bar{R}^{a} \leftarrow \bar{R}_{0}^{(n)}$

2. Compute $\bar{B}, \bar{c}, B_{m}$ and $B_{M}$ for $\bar{R}^{a}$ by the iterators

3. $\min B_{m_{\text {ini }}} \leftarrow B_{m}, \min B_{M_{\text {ini }}} \leftarrow B_{M}$

4. Mpaths $\leftarrow N(N-1) / 2, z_{\mathrm{APR}} \leftarrow 1$

5. While(1) (Mpaths $>0) \underline{\text { Do }}$

(a) Ncycle $\leftarrow 2$

(b) Npaths $\leftarrow$ Mpaths

(c) $\bar{R}^{a} \leftarrow \bar{R}_{0}^{(n)}$

(d) Compute $\bar{B}, \bar{c}, B_{m}$ and $B_{M}$ for $\bar{R}^{a}$ by the iterators

(e) $\min B_{m} \leftarrow B_{m}$

(f) While(2) (Npaths $>0)$ Do

i Search for the Npaths with lower $\xi(f)$

ii Compute with MMRA new paths for the corresponding $\mathrm{O}-\mathrm{D}$ pairs and define a new set of alternative paths for the network $-\bar{R}^{a}$, using $z_{\mathrm{APR}}$

iii Compute the new $\bar{B}, \bar{c}, B_{m}$ and $B_{M}$ by the iterators

iv If $\left(B_{m}<\min B_{m_{\mathrm{ini}}}\right.$ and $\left.B_{M}<\min B_{M_{\mathrm{ini}}}\right)$ Then

(A) $\min \mathrm{B}_{\mathrm{M}_{\text {ini }}} \leftarrow \mathrm{B}_{\mathrm{M}}, \min _{\mathrm{m}_{\text {ini }}} \leftarrow \mathrm{B}_{\mathrm{m}}$ (which means that the last obtained solution dominates the initial one for the considered network performance metrics)
(B) $\bar{R}^{*} \leftarrow \bar{R}^{a}$

$\mathrm{v}$ If $\left(B_{m}<\min B_{m}\right)$ Then

(A) $\min B_{m} \leftarrow B_{m}$ (which means that Npaths does not change in this case)

vi Else

(A) Npaths $\leftarrow$ Npaths -1

(B) If (Npaths $=0$ and Ncycle =2) Then

Ncycle $\leftarrow$ Ncycle -1

Npaths $\leftarrow N(N-1) / 2$

(C) Compute $\bar{B}, \bar{c}, B_{m}$ and $B_{M}$ for $\bar{R}^{a}$ by the iterators

(D) If (Npaths $<10$ and Ncycle $=1$ and Mpaths $\left.<M_{\text {sup }}\right)$ Then

$z_{\mathrm{APR}} \leftarrow$ Npaths $* 0.1$

(E) Else $z_{\mathrm{APR}} \leftarrow 1$

(End of While(2))

(g) Mpaths $\leftarrow$ Mpaths -1

(End of While(1))

6. $\bar{R}_{0}^{(n+1)} \leftarrow \bar{R}^{*}$ (set of alternative paths selected for the network in this path update cycle).

\subsection{Further improvements in $M O D R$}

In the initial version of MODR [9] the boundary values of the priority regions of MMRA (associated with "soft" constraints of the objective functions in $\mathscr{P}^{(2)}$ ) which represents acceptable and required values for the two path metrics were obtained from reference networks engineered for standard global network blocking probabilities in nominal and overload conditions; the changes in the preference regions would only result from alterations in the ideal solution $\left(O p^{1}, O p^{2}\right)$. A more flexible and effective scheme of boundary value specification is now introduced. Let:

$B_{\mathrm{av}}=\frac{1}{|L|} \sum_{l_{k} \in L} B_{k}, \quad c_{\mathrm{av}}=\frac{1}{|L|} \sum_{l_{k} \in L} c_{k}$, 
$\Delta B_{k}=\frac{B_{\mathrm{av}}-\min \left\{B_{k}\right\}}{2}, \quad \Delta c_{k}=\frac{c_{\mathrm{av}}-\min \left\{c_{k}\right\}}{2}$,

$B_{k}^{+}=B_{\mathrm{av}}+\Delta B_{k}, \quad B_{k}^{-}=B_{\mathrm{av}}-\Delta B_{k}$,

$c_{k}^{+}=c_{\mathrm{av}}+\Delta c_{k}, \quad c_{k}^{-}=c_{\mathrm{av}}-\Delta c_{k}$.

Then the required and acceptable values for the two path metrics $z^{1}$ (implied cost) and $z^{2}$ (blocking probability) are

$C_{\text {req }}=D c_{k}^{-}, \quad C_{\text {acc }}=D c_{k}^{+}$,

$B_{\text {req }}=1-\left(1-B_{k}^{-}\right)^{D}, \quad B_{\text {acc }}=1-\left(1-B_{k}^{+}\right)^{D}$,

where $D$ is the number of arcs of the alternative routes ( $D=2$ in our case). The main advantage of this scheme, confirmed by extensive experimentation, is the fact that it enables the priority region boundaries to adapt dynamically to different overload situations thereby overcoming the rigidity of the previous bounds which may lead in many overload situations to less efficient solutions from the point of view of global network performance. Overall the solutions obtained with this scheme tend to be more efficient than the previous ones, since the varying boundaries reflect the current situation of the links as a result of the updates of $B_{k}$ and $c_{k}$ performed in each iteration of the heuristic.

\section{Network performance of MODR-1}

In order to evaluate the performance of the MODR method incorporating the proposed heuristic of synchronous route calculation (designated hereafter as MODR-1) extensive computational experimentation was carried out, using three test networks: the network in [19] widely used in studies of dynamic routing method evaluation (network $\mathrm{M}$ for short) and two other networks with the same topology (six nodes, fully meshed) designated as network B and A. Network B was obtained by recalculating the arc capacities of network $M$ (while maintaining the same matrix of nominal traffic offered $\bar{A}_{0}$ ), with a standard dimensioning method for dynamic routing cir- cuit-switched networks [5]. Note that network M has strong asymmetries in many arc capacities, with respect to the direct traffic offered to them. Network A has a different matrix of nominal traffic offered with a smaller variation in traffic intensities than in network $\mathrm{B}$ and $\mathbf{M}$, and its arc capacities were calculated as in network B. The characteristics of each of these networks, including the initial route set $\bar{R}_{t_{0}}$ computed by the mentioned method [5], are shown in Appendix A. For assessing the potential of MODR-1 in terms of global network performance a comparative study with a known reference in dynamic routing, the RTNR method (Real Time Network Routing [2-4]) developed by AT\&T, was performed for the three mentioned networks. Note that the RTNR method is well known for its efficiency and remarkable network performance under overload conditions, largely resulting from the extensive use of very sophisticated hierarchical and dynamically adaptive service protection mechanisms, able of quickly and effectively responding to link overloading, traffic intensity fluctuations and degradation of node-to-node blocking probabilities. Results of global network performance measured by $B_{m}$ and $B_{M}$ are presented in Tables $1-3$ for the three test networks and different overload factors; the better values for each network metric are indicated in bold. The simulation results for MODR-1 and RTNR were obtained by a discrete-event simulator developed with a OMNET++ simulation platform and are the mid points of $95 \%$ confidence intervals obtained by the method of independent replications.

The following main conclusions may be drawn from these simulation results (and other results not presented here): (i) the heuristic of path selection beyond stabilising the final solution $\bar{R}_{t}$, in each updating period, enabled improved solutions from a global network performance point of view, as compared with most of the solutions (e.g. the solutions in [18] for network B) which might be obtained without the heuristic; (ii) excepting for the case of the poorly engineered network $\mathrm{M}$ for low and moderate overload (where $B_{m}$ and $B_{M}$ were in general very low and even below standardised requested values) and for very low blocking in network A and B, MODR-1 with $A P R$ performed 
Table 1

Global network performance for network $\mathrm{M}$

\begin{tabular}{|c|c|c|c|c|c|c|}
\hline \multirow[t]{3}{*}{ Overl. factor } & \multicolumn{4}{|c|}{ MODR-1 } & \multicolumn{2}{|l|}{ RTNR } \\
\hline & \multicolumn{2}{|c|}{$\begin{array}{l}\text { Analytical } \\
\text { model }\end{array}$} & \multicolumn{2}{|l|}{ Simulation model } & \multicolumn{2}{|l|}{ Simulation model } \\
\hline & $B_{m}$ & $B_{M}$ & $B_{m} \pm \Delta$ & $B_{M} \pm \Delta$ & $B_{m} \pm \Delta$ & $B_{M} \pm \Delta$ \\
\hline $0 \%$ & $<10^{-3}$ & 0.001 & $<10^{-3}$ & $(4.0 \pm 6.0) \times 10^{-3}$ & $<10^{-3}$ & $<10^{-3}$ \\
\hline $10 \%$ & 0.001 & 0.009 & $(3.0 \pm 0.18) \times 10^{-3}$ & $(2.2 \pm 0.15) \times 10^{-2}$ & $(1.0 \pm 0.11) \times 10^{-3}$ & $(5.0 \pm 1.1) \times 10^{-3}$ \\
\hline $20 \%$ & 0.005 & 0.035 & $(9.0 \pm 0.21) \times 10^{-3}$ & $(5.8 \pm 0.16) \times 10^{-2}$ & $(4.0 \pm 0.30) \times 10^{-3}$ & $(2.5 \pm 0.24) \times 10^{-2}$ \\
\hline $30 \%$ & 0.019 & 0.076 & $(2.8 \pm 0.13) \times 10^{-2}$ & $(1.05 \pm 0.031) \times 10^{-1}$ & $(2.7 \pm 0.15) \times 10^{-2}$ & $(1.44 \pm 0.13) \times 10^{-1}$ \\
\hline $40 \%$ & 0.060 & 0.138 & $(6.3 \pm 0.15) \times 10^{-2}$ & $(1.76 \pm 0.06) \times 10^{-1}$ & $(6.3 \pm 0.16) \times 10^{-2}$ & $(2.57 \pm 0.055) \times 10^{-1}$ \\
\hline $50 \%$ & 0.102 & 0.174 & $(1.01 \pm 0.018) \times 10^{-1}$ & $(2.62 \pm 0.063) \times 10^{-1}$ & $(1.01 \pm 0.018) \times 10^{-1}$ & $(3.35 \pm 0.033) \times 10^{-1}$ \\
\hline $60 \%$ & 0.133 & 0.361 & $(1.37 \pm 0.013) \times 10^{-1}$ & $(3.41 \pm 0.059) \times 10^{-1}$ & $(1.38 \pm 0.015) \times 10^{-1}$ & $(3.97 \pm 0.037) \times 10^{-1}$ \\
\hline $70 \%$ & 0.167 & 0.398 & $(1.69 \pm 0.015) \times 10^{-1}$ & $(4.16 \pm 0.022) \times 10^{-1}$ & $(1.73 \pm 0.017) \times 10^{-1}$ & $(4.46 \pm 0.029) \times 10^{-1}$ \\
\hline $80 \%$ & 0.202 & 0.469 & $(2.02 \pm 0.012) \times 10^{-1}$ & $(4.54 \pm 0.028) \times 10^{-1}$ & $(2.04 \pm 0.016) \times 10^{-1}$ & $(4.79 \pm 0.014) \times 10^{-1}$ \\
\hline
\end{tabular}

Table 2

Global network performance for network B

\begin{tabular}{|c|c|c|c|c|c|c|}
\hline \multirow[t]{3}{*}{ Overl. factor } & \multicolumn{4}{|c|}{ MODR-1 } & \multicolumn{2}{|l|}{ RTNR } \\
\hline & \multicolumn{2}{|c|}{$\begin{array}{l}\text { Analytical } \\
\text { model }\end{array}$} & \multicolumn{2}{|l|}{ Simulation model } & \multicolumn{2}{|l|}{ Simulation model } \\
\hline & $B_{m}$ & $B_{M}$ & $B_{m} \pm \Delta$ & $B_{M} \pm \Delta$ & $B_{m} \pm \Delta$ & $B_{M} \pm \Delta$ \\
\hline $0 \%$ & 0.005 & 0.015 & $(9.0 \pm 0.43) \times 10^{-3}$ & $(2.0 \pm 0.37) \times 10^{-2}$ & $(7.0 \pm 0.67) \times 10^{-3}$ & $(2.9 \pm 0.64) \times 10^{-2}$ \\
\hline $10 \%$ & 0.056 & 0.077 & $(5.9 \pm 0.12) \times 10^{-2}$ & $(9.6 \pm 0.66) \times 10^{-2}$ & $(5.8 \pm 0.11) \times 10^{-2}$ & $(1.80 \pm 0.097) \times 10^{-1}$ \\
\hline $20 \%$ & 0.113 & 0.136 & $(1.16 \pm 0.011) \times 10^{-1}$ & $(1.54 \pm 0.064) \times 10^{-1}$ & $(1.11 \pm 0.013) \times 10^{-1}$ & $(2.57 \pm 0.12) \times 10^{-1}$ \\
\hline $30 \%$ & 0.165 & 0.193 & $(1.70 \pm 0.014) \times 10^{-1}$ & $(1.93 \pm 0.021) \times 10^{-1}$ & $(1.93 \pm 0.021) \times 10^{-1}$ & $(2.96 \pm 0.038) \times 10^{-1}$ \\
\hline $40 \%$ & 0.214 & 0.246 & $(2.18 \pm 0.013) \times 10^{-1}$ & $(2.47 \pm 0.022) \times 10^{-1}$ & $(2.16 \pm 0.012) \times 10^{-1}$ & $(3.15 \pm 0.077) \times 10^{-1}$ \\
\hline
\end{tabular}

Table 3

Global network performance for network A

\begin{tabular}{|c|c|c|c|c|c|c|}
\hline \multirow[t]{3}{*}{ Overl. factor } & \multicolumn{4}{|c|}{ MODR-1 } & \multirow{2}{*}{\multicolumn{2}{|c|}{$\frac{\text { RTNR }}{\text { Simulation model }}$}} \\
\hline & \multicolumn{2}{|c|}{$\begin{array}{l}\text { Analytical } \\
\text { model }\end{array}$} & \multicolumn{2}{|l|}{ Simulation model } & & \\
\hline & $\overline{B_{m}}$ & $B_{M}$ & $B_{m} \pm \Delta$ & $B_{M} \pm \Delta$ & $B_{m} \pm \Delta$ & $B_{M} \pm \Delta$ \\
\hline $0 \%$ & 0.004 & 0.006 & $(6 . \pm 0.70) \times 10^{-3}$ & $(1.3 \pm 0.16) \times 10^{-2}$ & $(3.0 \pm \mathbf{0 . 5 3}) \times 10^{-3}$ & $(6.0 \pm 1.5) \times 10^{-3}$ \\
\hline $10 \%$ & 0.030 & 0.055 & $(3.3 \pm 0.31) \times 10^{-2}$ & $(4.7 \pm 0.51) \times 10^{-2}$ & $(4.1 \pm 0.29) \times 10^{-2}$ & $(6.1 \pm 0.44) \times 10^{-2}$ \\
\hline $20 \%$ & 0.079 & 0.123 & $(8.0 \pm 0.28) \times 10^{-2}$ & $(1.04 \pm 0.063) \times 10^{-1}$ & $(9.0 \pm 0.27) \times 10^{-2}$ & $(1.33 \pm 0.089) \times 10^{-1}$ \\
\hline $30 \%$ & 0.118 & 0.177 & $(1.30 \pm 0.028) \times 10^{-1}$ & $(1.59 \pm 0.073) \times 10^{-1}$ & $(1.29 \pm 0.022) \times 10^{-1}$ & $(1.86 \pm 0.087) \times 10^{-1}$ \\
\hline $40 \%$ & 0.158 & 0.242 & $(1.74 \pm 0.023) \times 10^{-1}$ & $(2.14 \pm 0.066) \times 10^{-1}$ & $(1.67 \pm 0.018) \times 10^{-1}$ & $(2.26 \pm 0.11) \times 10^{-1}$ \\
\hline
\end{tabular}

better than RTNR - in fact the solutions of MODR-1 either dominate the RTNR solutions or are non-dominated with respect to the latter, cases in which they enable a reduction in maxi- mum marginal blocking probability at the cost of a light increase in network mean blocking probability; (iii) only for low or very low overload where even so MODR-1 values for $B_{m}$ are normally 
below typical required values (e.g. $\leqslant 0.5 \%$ at $0 \%$ overload), RTNR tends to give better results than MODR-1 in terms of $B_{m}$.

Also note some discrepancies between the analytical and simulation results for MODR-1, specially important for low overload where the analytical model underestimates the values of the parameters $B_{m}$ and $B_{M}$. The major factor that might explain these errors, beyond the intrinsic numerical imprecision of the analytical solution and the uncertainty in the simulation results (particularly important for very low blocking, where even for many hundreds of thousands of simulated calls a "rare event" is at stake), has to do with the assumed simplifications in the stochastic traffic model of the links (superposition of independent Poisson traffic flows and independent occupations in the path links). Note that this simplification, which is generally assumed in the analytical models of dynamic routing methods, is necessary to cope with the inherent complexity of the model, which otherwise could easily become intractable. In theory one should use more realistic representations of the traffic flows namely based on their mean and variance, enabling to obtain better estimates of the blocking probabilities (without the biasing towards underestimation for zero or low overload) obtained from known models in teletraffic theory, such as in [8]. In any case this, or other eventually more realistic traffic representations based on higher order moments, would turn the analytical model easily intractable, specially having in mind that it has to be solved numerically a great number of times in the context of the heuristic of synchronous path selection.

Overall these results seem very encouraging with respect to the potential of MODR-1 in terms of global network performance, specially when the preservation of the quality of service of more "sensitive" traffic flows of low intensity is an important concern.

\section{Conclusions and further work}

In order to cope with the complexity of a bidimensional alternative dynamic routing method for circuit switched telecommunications networks and with its effects in terms of network performance a complete analytical model has been presented. This model enabled to make it explicit an instability problem in the bi-objective synchronous path calculation procedure, previously detected, and to express a bi-dimensional compromise dynamic routing problem at the global network level seeking the minimisation of the network mean blocking probability and the maximal marginal node-to-node blocking probability. This instability problem of an oscillatory type is associated with the complex non-linear interdependencies between the coefficients of the two objective functions of an underlying bi-objective shortest path problem, with the interdependencies between both sets of coefficients and the computed paths and from the discrete nature of the model. Also a heuristic of synchronous path selection, enabling to overcome that instability problem and select "good" compromise solutions in terms of the two global network performance criteria, was formalised.

Finally the relative performance of the routing method using the proposed heuristic procedure was analysed by recurring to a discrete event simulation platform enabling to compare the global network performance criteria values of the model incorporating the heuristic, with those obtained with a reference dynamic routing method RTNR (Real-Time Network Routing) developed by AT\&T, for some test networks. Globally the network performance obtained with the model is quite satisfactory, namely for moderate and high overloads where it performs tendentially better than RTNR. Note that these are working conditions where the effectiveness of any dynamic routing method is of greater relevance. Also the explicit incorporation in the routing model of a second metric associated with the maximal marginal node-to-node call blocking probability seems to be an "added-value" of this approach, namely in situations where the protection against an excessive degradation of marginal blocking probabilities associated with small intensity traffic flows may be a particular concern. The major limitation of the method under analysis is related with its inherent complexity and the computational processing cost associated with the necessity of repetitive numerical resolution of the analytical 
Table 4

Test networks

\begin{tabular}{|c|c|c|c|c|c|c|c|c|c|}
\hline \multirow[t]{2}{*}{ O-D pair } & \multicolumn{3}{|c|}{ Network A } & \multicolumn{3}{|c|}{ Network B } & \multicolumn{3}{|c|}{ Network M } \\
\hline & $\begin{array}{l}\text { Link } \\
\text { capac. }\end{array}$ & $\begin{array}{l}\text { Offer. } \\
\text { traff. }\end{array}$ & $\begin{array}{l}\text { Intermed. } \\
\text { node }\end{array}$ & $\begin{array}{l}\text { Link } \\
\text { capac. }\end{array}$ & $\begin{array}{l}\text { Offer. } \\
\text { traff. }\end{array}$ & $\begin{array}{l}\text { Intermed. } \\
\text { node }\end{array}$ & $\begin{array}{l}\text { Link } \\
\text { capac. }\end{array}$ & Offer. & $\begin{array}{l}\text { Intermed } \\
\text { node }\end{array}$ \\
\hline $1-2$ & 36 & 27 & 3 & 41 & 27.47 & 3 & 36 & 27.47 & 3 \\
\hline $1-3$ & 13 & 6 & 4 & 13 & 6.97 & 4 & 24 & 6.97 & 5 \\
\hline $1-4$ & 33 & 25 & 5 & 276 & 257.81 & 5 & 324 & 257.81 & - \\
\hline $1-5$ & 27 & 20 & 6 & 33 & 20.47 & 6 & 48 & 20.47 & 3 \\
\hline $1-6$ & 31 & 20 & 2 & 46 & 29.11 & 2 & 48 & 29.11 & 5 \\
\hline $2-3$ & 29 & 25 & 4 & 30 & 25.11 & 4 & 96 & 25.11 & - \\
\hline $2-4$ & 17 & 10 & 5 & 112 & 101.61 & 5 & 96 & 101.61 & 3 \\
\hline $2-5$ & 37 & 30 & 6 & 88 & 76.78 & 6 & 108 & 76.78 & 3 \\
\hline $2-6$ & 25 & 20 & 1 & 94 & 82.56 & 1 & 96 & 82.56 & 3 \\
\hline $3-4$ & 17 & 11 & 5 & 18 & 11.92 & 5 & 12 & 11.92 & 1 \\
\hline $3-5$ & 14 & 8 & 6 & 12 & 6.86 & 6 & 48 & 6.86 & 6 \\
\hline $3-6$ & 19 & 13 & 1 & 21 & 13.25 & 1 & 24 & 13.25 & 2 \\
\hline $4-5$ & 13 & 9 & 6 & 88 & 79.42 & 6 & 192 & 79.42 & 1 \\
\hline 4-6 & 27 & 20 & 1 & 94 & 83.0 & 1 & 84 & 83.0 & 5 \\
\hline $5-6$ & 18 & 12 & 1 & 137 & 127.11 & 1 & 336 & 127.11 & - \\
\hline
\end{tabular}

model. Further work is also taking place concerning the extension of MODR-1 formulation and implementation to multi-service networks, including networks based on technologies ATM or MPLS (for connection-oriented services), based on an appropriate generalisation of the concept of implied cost and adequate multiclass traffic models, associated with relevant quality of service (traffic dependent) metrics. Finally the "tuning" of important parameters of the method, namely the path updating period and service protection mechanism parameters, will have to be tackled through extensive use of the simulation test-bed.

\section{Acknowledgements}

We thank Luísa Jorge for her assistance in the development of the simulation test-bed and Tiago Sá for the implementation of the network design model based on the algorithm [5].

\section{Appendix A. Test networks}

The characterization of networks $\mathrm{A}, \mathrm{B}$, and $\mathrm{M}$ including $\bar{C}, \bar{A}_{0}$ (nominal load) and the intermediate nodes of the routes in $\bar{R}_{0}^{(1)}$ is in Table 4 .

\section{References}

[1] C. Henggeler Antunes, J. Craveirinha, J. Clímaco, C. Barrico, A multiple objective routing algorithm for integrated communication networks, in: P. Key, D. Smith (Eds.), ITC-16 Teletraffic Engineering in a Competitive World, vol. 3b, Elsevier Science B.V., Amsterdam, 1999, pp. 1291-1300.

[2] G.R. Ash, An analytical model for adaptative routing networks, IEEE Transactions on Communications 41 (11) (1993) 1748-1759.

[3] G.R. Ash, Dynamic network evolution, with examples from AT\&T's evolving dynamic network, IEEE Communications Magazine (1995) 26-39.

[4] G.R. Ash, Dynamic Routing in Telecommunications Networks, McGraw-Hill, New York, 1998.

[5] G.R. Ash, R.H. Cardwell, R.P. Murray, Design and optimization of networks with dynamic routing, Bell Systems Technical Journal 60 (8) (1981) 17871820 .

[6] D. Bertsekas, R. Gallager, Data Networks, Prentice-Hall, Englewood Cliffs, NJ, 1992.

[7] J. Clímaco, E.Q. Martins, A bicriterion shortest path algorithm, European Journal of Operational Research (11) (1982) 399-404.

[8] J. Craveirinha, T. Gomes, S. Esteves, L. Martins, A method for calculating marginal variances in teletraffic networks with multiple overflows, in: J. Janssen, S. Osaki (Eds.), Proceedings of the First Euro-Japanese Workshop on Stochastic Risk Modelling for Finance, Insurance, Production and Reliability, vol. II, Brussels, 1998.

[9] J. Craveirinha, L. Martins, T. Gomes, C.H. Antunes, J. Clímaco, A new multiple objective dynamic routing 
method using implied costs, Journal of Telecommunications and Information Technology (3) (2003) 50-59.

[10] H.M. Elsayed, M.S. Mahmoud, A.Y. Bilal, J. Bernussou, Adaptive alternate-routing in telephone networks: Optimal and equilibrium solutions, Information and Decision Technologies (14) (1988) 65-74.

[11] R.J. Gibbens, F.P. Kelly, Dynamic routing in fully connected networks, IMA Journal of Mathematical Control and Information 7 (1990) 77-111.

[12] A. Girard, Routing and Dimensioning in Circuit-Switched Networks, Addison-Wesley Publishing Company, Reading, MA, 1990.

[13] T. Gomes, L. Martins, J.F. Craveirinha, An efficient algorithm for calculating $k$ shortest paths with a maximum number of arcs, Investigação Operacional 1 (21) (2001) 235-244.

[14] F.P. Kelly, Blocking probabilities in large circuit-switched networks, Advances in Applied Probability 18 (1986) 473 505.

[15] F.P. Kelly, Routing in circuit-switched networks: Optimization, shadow prices and decentralization, Advances in Applied Probability 20 (1988) 112-144.

[16] F.P. Kelly, Routing and capacity allocation in networks with trunk reservation, Mathematics of Operations Research 15 (4) (1990) 771-793.

[17] E.Q.V. Martins, M.M.B. Pascoal, J.L.E. Santos, Deviation algorithms for ranking shortest paths, International Jour- nal of Foundations of Computer Science 10 (1999) 247263.

[18] L. Martins, J. Craveirinha, J. Clímaco, T. Gomes, Implementation and performance of a new multiple objective dynamic routing method for multiexchange networks, Journal of Telecommunications and Information Technology (3) (2003) 60-66.

[19] Debasis Mitra, Judith B. Seery, Comparative evaluation of randomized and dynamic routing strategies for circuitswitched networks, IEEE Transactions on Communications 39 (1) (1991) 102-116.

[20] C. Pornavalai, G. Chakraborty, N. Shiratori, Routing with multiple QoS requirements for supporting multimedia applications, Telecommunication System 9 (1998) 357373.

[21] J.M. Rodrigues, J.C. Clímaco, J.R. Current, An interactive bi-objective shortest path approach: Searching for unsupported nondominated solutions, Computers and Operations Research 26 (1999) 789-798.

[22] R. Vogel, R.G. Herrtwich, W. Kalfa, H. Wittig, L.C. Wolf, QoS-based routing of multimedia streams in computer networks, IEEE Journal on Selected Areas in Communications 14 (7) (1996) 1235-1244.

[23] Z. Wang, J. Crowcroft, Quality-of-service routing for supporting multimedia applications, IEEE Journal on Selected Areas in Communications 14 (7) (1996) 12281234. 